\title{
A Mixed-Methods Study of the Technical Feasibility and Patient Acceptability of a Real-Time Adherence Monitor in Breast Cancer Survivors Taking Adjuvant Endocrine Therapy
}

\section{Emma E. Bright ( $\square$ emma.bright@colorado.edu )}

University of Colorado Boulder https://orcid.org/0000-0001-6682-4561

Sarah R. Genung

University of Colorado Boulder

Annette L Stanton

University of California Los Angeles

Joanna J. Arch

University of Colorado Boulder

\section{Research Article}

Keywords: Breast cancer, medication adherence, endocrine therapy, real-time adherence monitor

Posted Date: December 6th, 2021

DOl: https://doi.org/10.21203/rs.3.rs-974314/v1

License: (c) (1) This work is licensed under a Creative Commons Attribution 4.0 International License.

Read Full License 


\section{Abstract}

Purpose: Oral anti-cancer medications are increasingly common and endocrine therapies represent the most common oral anti-cancer medications in breast cancer. Adjuvant endocrine therapies reduce the likelihood of recurrence and mortality in the approximately $80 \%$ of women diagnosed with hormonereceptor positive breast cancer, thus rendering adherence essential. Real-time medication adherence monitors, such as the Wisepill electronic pillbox, transmit adherence data remotely, allowing for early intervention for non-adherence. However, their feasibility and acceptability have yet to be examined among breast cancer survivors taking endocrine therapies.

Methods: This study presents quantitative patient-report and technical support data and qualitative patient acceptability data on using Wisepill, a common real-time adherence monitor, among 88 breast cancer survivors prescribed adjuvant endocrine therapy.

Results: This mixed-methods study of a common real-time adherence monitor, among the first in breast cancer survivors taking adjuvant endocrine therapy, demonstrates its technical feasibility and patient acceptability.

Conclusion: The use of wireless medication monitors that transmit real-time adherence data are uniquely promising for maximizing the benefits of adjuvant endocrine therapy by allowing for continuous tracking, ongoing communication with oncologic or research teams, and early intervention. This study demonstrates the feasibility and patient acceptability of one such real-time adherence monitor.

\section{Background}

Oral anti-cancer medications are increasingly common [1]; however, medication adherence (i.e., taking the medication in the dose, frequency and duration as prescribed) among adults with cancer remains suboptimal [2-4]. Nonadherence is associated with poor treatment efficacy, greater health care costs, and increased hospital utilization among patients with cancer [4]. Medication adherence is a key pathway through which oncologic practice is translated into patient outcomes [5]. A common class of oral anticancer medication is adjuvant endocrine therapy (AET), adjuvant medications that reduce recurrence and mortality among the approximately $80 \%$ of breast cancer survivors with hormone-receptor positive tumors [6-8]. Recommendations suggest five to ten years of AET after primary treatment, making longterm adherence particularly challenging for breast cancer survivors [9]. Despite the benefits of adherence, a systematic review revealed that AET adherence after 5 years ranged from $41-72 \%$ [2]. The clear advantages of AET coupled with the challenges of adherence render the accurate assessment of adherence essential and early intervention for nonadherence necessary for optimizing medical outcomes among breast cancer survivors.

Current approaches for assessing medication adherence include indirect methods such as patient selfreport on questionnaires and interviews, pill counts, prescription records, and more direct methods, such as electronic medication monitors [10]. Electronic medication adherence monitors are precise and can 
track patterns in adherence over time without being subject to poor patient recall or social desirability. Advancements in electronic adherence monitors, such as the Wisepill real-time adherence monitor (Wisepill Technologies, Cape Town, South Africa), have eliminated the need for return visits to download device data and can remotely transmit adherence information in real time via wireless networks. Realtime adherence monitors allow for early intervention among patients who have discontinued their medications [11], as they detect lapses in adherence as they occur, providing novel opportunities to prevent treatment failure among breast cancer survivors taking AET.

In that real-time monitors represent the future of adherence assessment, it remains critical to evaluate their feasibility and acceptability in cancer populations that are commonly prescribed oral medication. Real-time monitoring of physical symptoms is well-established in many cancer care settings [12,13], however, real-time monitoring of medication adherence is not. Despite widespread use of medication adherence monitoring among adults with HIV/AIDS [14,15], the feasibility and acceptability of real-time medication adherence monitors such as Wisepill are not yet established among breast cancer survivors prescribed AET. The current study provides the first known published report on the use of real-time medication adherence-monitoring devices in breast cancer survivors. This study uses mixed methods to evaluate patient and technical support perspectives on Wisepill, a real-time monitor. Our patient acceptability and feasibility goals were that: (1) patients would find the real-time medication adherencemonitoring devices acceptable on both quantitative and qualitative measures, (2) 95\% of participants would complete the 30-day device feasibility baseline period, thus demonstrating patient feasibility of device use and (3) participants could complete the study with minimal contact from the research team to resolve technical issues.

\section{Methods}

\section{Participants}

Patients who were prescreened as medically eligible by electronic chart review were recruited from Rocky Mountain Cancer Centers in Colorado to participate in a values-based clinical intervention trial to promote AET adherence (clinical trial identifier: NCT03980093, findings from the values-based intervention are currently under review elsewhere). The study eligibility criteria included: diagnosed with Stage 0-III hormone receptor-positive breast cancer, completed primary cancer treatment, were prescribed AET in the past 2.5 years with at least 1 year remaining, and reported at least modest difficulty taking AET. Of the 977 mailings that were sent to prescreened patients, 207 women responded, 99 were eligible, 11 women were not interested in participating after hearing more detail about the study, and 88 women enrolled. All participants provided informed consent and the University of Colorado Boulder Institutional Review Board approved the study.

\section{Procedure}

Participants were mailed a Wisepill device, a wireless electronic pillbox that sends real-time data when opened, along with instructions for its use. The device consists of a portable black rectangular box (4.33" 
long $\times 1.77^{\prime \prime}$ wide $\times .47^{\prime \prime}$ deep) that holds up to 30 large or 60 small pills. The instructions included visuals, basic written instructions, and troubleshooting tips printed on a $5 \times 8$ " laminated card. After participants received the post-mailed package with the Wisepill and the instructions, a member of the research team called to confirm that the participants were able to set up and use the device. The devices were remotely monitored online by the research team several times per week to ensure proper functioning. The daily text reminder feature of Wisepill was disabled to focus on use of the device itself and the values intervention content.

\section{Measures}

Feasibility. Patient feasibility was assessed by examining the number of participants who successfully used the Wisepill device over the 30-day baseline period and the entirety of the seven-month study period.

Wisepill signal lapses greater than 48 hours were briefly investigated by a member of the research team via phone to determine if the cause was a device malfunction or behavioral. If the lack of recorded data was not behavioral, the nature of the technical difficulty and number of contacts needed to resolve it were recorded as a measure of technical feasibility.

Patient Acceptability. Breast cancer survivors rated on a 5-point Likert-type scale ( 1 not at all, 5 extremely) how helpful and convenient they found the Wisepill device, and how likely they were to recommend the device to another breast cancer survivor taking AET.

Qualitative information on patient acceptability of Wisepill was extracted from semi-structured phone exit interviews conducted with a subsample of participants $(n=38)$ by four trained post-baccalaureate interviewers. To ensure that the full range of experiences was represented in the interviews, 23 participants were randomly selected within satisfied, neutral, and dissatisfied strata based on their quantitative and qualitative responses to questions about their overall study satisfaction. As the responses to the values-based intervention were overwhelmingly positive, this approach resulted in oversampling negative responses; thus, 15 additional participants were randomly selected using a random number generator.

Interviews were transcribed and coded for both a priori and emergent codes regarding patient acceptability of Wisepill and suggestions for future Wisepill use. Example questions from the interviews include, "Did you experience any benefits in using the Wisepill box?" and "How convenient or inconvenient was it [Wisepill] to use?" Participants were also asked about how the monitoring function of the Wisepill may have affected their behavior and their recommendations for future use. All transcripts were coded in ATLAS.ti version 9.1 twice by 2 to 3 independent coders: once for broader themes and again for relevant sub-themes. After coding, the team met to establish consensus by resolving any conflicts through discussion.

\section{Results}




\section{Sample characteristics}

On average, participants were 58 years of age, 14 months from the end of primary treatment, and prescribed AET approximately 13 months prior to study entry (Table 1). The majority of participants were non-Latina white (92\%) and prescribed aromatase inhibitors (63\%).

Feasibility: Completion.All participants (100\%) completed a 30-day Wisepill monitoring baseline, exceeding the study's 95\% completion goal, and $98.86 \%$ (87/88) completed the six-month post-baseline Wisepill monitoring period.

Feasibility: Technical difficulties. Over the 7-month study, $13.64 \%$ of participants $(n=12)$ experienced technical difficulties using Wisepill which required a total of 38 contacts to resolve, including losing connectivity $(n=9$, contacts $=25)$, not registering pillbox openings $(n=1$, contacts $=6)$, charging cable not working $(n=1$, contacts $=4)$, and needing to reset the battery $(n=1$, contacts $=3)$. These contacts were almost exclusively initiated by the research team, specifically all those related to losing connectivity and not registering pill box openings were only research-team initiated.

Patient acceptability: Quantitative ratings. On a 5-point Likert-type scale, on average, participants found the Wisepill box to be moderately helpful $(M=3.15, S D=1.31)$, moderately convenient $(M=3.66, S D=$ $1.36)$, and were somewhat likely $(M=3.40, S D=1.49)$ to recommend the device to someone else taking AET.

Patient acceptability: Qualitative interviews.Women responded to open-ended prompts about the acceptability of Wisepill and offered suggestions for future use. Most breast cancer survivors found Wisepill convenient to use $(n=27)$. For example, when asked about the convenience of the Wisepill, one participant stated:

It was not at all inconvenient. It's no different than a pill bottle to me in terms of use. (1056)

Many women $(n=22)$ also found benefits in using the Wisepill (even though the daily reminder feature was inactive). Breast cancer survivors found the real-time tracking features of the Wisepill to be a source of motivation, for example, one participant stated:

I think the idea that someone was tracking you and that they were going to ask you about it... was a little more motivating to make sure I took it. (1046)

The mere presence of the device was a helpful reminder and benefit for others:

It made me aware of every day that I was taking my medications. And it helped me keep track of them. (1068)

While others did not perceive any benefits from their use of Wisepill, they did note the ease of use: 
Did I experience any benefits in using it? No, it was convenient to have. It was easy to open instead of opening a pill bottle, so that was kind of nice, you just kind of slid it flipped it open. (1073)

Many participants $(n=16)$ reported no changes in their behavior or motivation to take AET because their adherence was being monitored. For example, when asked whether knowing that her adherence data were being monitored had changed her behavior, one participant stated:

I really don't think it affected it at all. Because yeah, I just don't think that that box motivated me in any way to take my medications. I take them because they're prescribed... (1066)

Other participants $(n=9)$ reported positively changing their adherence behavior or feeling more motivated or committed to taking AET because of the knowledge that Wisepill was remotely transmitting their adherence data. One participant stated:

I think occasionally like I said, I don't want to take it today. And then knowing that somebody was going to know whether I took it or not, okay fine I'll take it. (1037)

Participants were also asked for their recommendations for future use of the Wisepill device. Suggestions included daily access to an online Wisepill medication $\log (n=10)$, a device-initiated reminder (alarm, blinking light, text message) to take their medication $(n=9)$, and a 7-day internal divider to track their medication ( $n=11$; Table 2).

\section{Discussion}

Findings demonstrate that real-time wireless monitoring of AET adherence among breast cancer survivors is generally acceptable to patients and technically feasible. That $100 \%$ of participants completed a 30-day Wisepill monitoring baseline also provides strong evidence of feasibility. The most common technical issue, lost wireless connectivity, delayed the transmission of data but still allowed for adherence information to be transmitted once the device reconnected. Although technical components of the device must be monitored carefully to ensure proper functioning, serious technical issues were rare. These findings converge with previous research on the technical feasibility of Wisepill among patients with HIV/AIDS [14,16].

In addition, the devices were acceptable to patients, even with some of the more useful features, such as reminders, inactivated to focus on the use of the device itself and the values intervention content. The devices were rated as moderately helpful, convenient, and recommendable on quantitative measures. In qualitative exit interviews, many breast cancer survivors reported that the devices were convenient and beneficial for adherence. The accountability that comes from the devices being monitored was noted as a benefit by some participants, while other participants found the mere presence of the device to be a helpful reminder to take their medications. The ability of standard electronic adherence monitoring devices to serve as a visual reminder has been noted as a facilitator of medication adherence among 
patients with HIV/AIDS [17]. While not all participants found benefits in the use of Wisepill, many breast cancer survivors did note its convenience and ease of use.

For future use, patients had consistent and feasible recommendations including a daily pill log, daily reminders, and a pillbox divider, which could be incorporated into future interventions or clinic policies to promote adherence. Wisepill also supports a reminder function and can be used with a 7-day pillbox divider, as recommended by the current sample.

This study's primary limitation is the lack of racial and ethnic diversity in the sample, which requires caution in generalizing the findings to diverse groups. In addition, the full capabilities of the Wisepill device, namely the reminder function was not enabled in the present study, similar to studies in HIV/AIDS populations [18]. However, it is notable that participants still found the devices to largely be acceptable despite the absence of the reminder function. Research has shown some minimal benefit of electronic medication device reminders alone for promoting adherence among patients with non-cancer illnesses [19]. Thus, future research examining the patient acceptability of real-time adherence devices with all features activated is warranted.

Research has shown that AET non-adherence predicts breast cancer recurrence and subsequent mortality $[3,4,8,20]$. More accurate adherence monitoring, in combination with the potential for early intervention for non-adherence, would allow for prioritization of limited resources to promote adherence among those at greatest risk. The use of wireless medication monitors that transmit real-time adherence data are uniquely promising for maximizing the benefits of AET by allowing for continuous tracking, ongoing communication with oncologic or research teams, and early intervention. The present study is the first to our knowledge to demonstrate the technical feasibility and patient acceptability of a real-time wireless medication adherence monitor among breast cancer survivors taking AET, thus establishing a foundation for further use and investigation of these promising devices in this population.

\section{Declarations}

\section{Ethics approval}

University of Colorado Institutional Review Board approval was obtained for this study.

\section{Consent to participate}

Informed consent was obtained from all participants.

\section{Consent for publication}

Not applicable.

\section{Availability of data and material}


Upon reasonable request, the quantitative data analyzed in this study are available from the corresponding author in accordance with institutional policies. Qualitative data currently lack widely accepted de-identification standards.

\section{Authors' contributions}

All authors have made substantial contributions to the conception and design of the work, including the acquisition, analysis, and interpretation of data, as well as drafting the manuscript. All authors have approved the current version of manuscript and are accountable for all aspects of the work.

\section{Acknowledgments and Funding Information}

This work was supported by a grant from the National Cancer Institute, R21CA218723 (PI: Arch) and the Breast Cancer Research Foundation, BCRF-21-153 (PI: Stanton).

\section{Conflicts of interest/Competing interests}

The authors have no relevant financial or non-financial interests to disclose.

\section{References}

1. Weingart SN, Brown E, Bach PB et al. NCCN Task Force Report: Oral Chemotherapy. JNCCN 2008;6(S3):S-S114

2. Murphy CC, Bartholomew LK, Carpentier MY, Bluethmann SM, Vernon SW (2012) Adherence to adjuvant hormonal therapy among breast cancer survivors in clinical practice: a systematic review. Breast Cancer Res Treat 134(2):459-478

3. Ruddy K, Mayer E, Partridge A (2009) Patient adherence and persistence with oral anticancer treatment. CA: A Cancer Journal for Clinicians 59(1):56-66

4. Partridge AH, Avorn J, Wang PS, Winer EP (2002) Adherence to therapy with oral antineoplastic agents. J Natl Cancer Inst 94(9):652-661

5. Kravitz RL, Melnikow J (2004) Medical adherence research: time for a change in direction? Med Care 42(3):197-199

6. Dunnwald LK, Rossing MA, Li Cl (2007) Hormone receptor status, tumor characteristics, and prognosis: a prospective cohort of breast cancer patients. Breast Cancer Res 9(1):R6

7. Makubate B, Donnan PT, Dewar JA, Thompson AM, McCowan C (2013) Cohort study of adherence to adjuvant endocrine therapy, breast cancer recurrence and mortality. $\mathrm{Br} J$ Cancer 108(7):1515-1524

8. Hershman DL, Kushi LH, Shao T et al (2010) Early discontinuation and nonadherence to adjuvant hormonal therapy in a cohort of 8,769 early-stage breast cancer patients. J Clin Oncol 28(27):41204128

9. Burstein HJ, Temin S, Anderson $\mathrm{H}$ et al (2014) Adjuvant endocrine therapy for women with hormone receptor-positive breast cancer: American society of clinical oncology clinical practice guideline 
focused update. J Clin Oncol 32(21):2255-2269

10. Osterberg L, Blaschke T (2005) Adherence to medication. NEJM 353(5):487-497

11. Haberer JE (2017) Actionable adherence monitoring to optimise intervention. Lancet HIV 4(1):e5-e6

12. Kofoed S, Breen S, Gough K, Aranda S (2012) Benefits of remote real-time side-effect monitoring systems for patients receiving cancer treatment. Oncol Rev 6(1):e7

13. Warrington L, Absolom K, Conner M et al (2019) Electronic systems for patients to report and manage side effects of cancer treatment: systematic review. J Med Internet Res 21(1):e10875

14. Haberer JE, Kahane J, Kigozi I et al (2010) Real-time adherence monitoring for HIV antiretroviral therapy. AIDS Behav 14(6):1340-1346

15. Evans D, Berhanu R, Moyo F, Nguweneza A, Long L, Fox MP (2016) Can short-term use of electronic patient adherence monitoring devices improve adherence in patients failing second-line antiretroviral therapy? Evidence from a pilot study in Johannesburg, South Africa. AIDS Behav 20(11):2717-2728

16. Bachman DeSilva M, Gifford AL, Keyi X et al. Feasibility and acceptability of a real-time adherence device among HIV-positive IDU patients in China. AIDS research and treatment. 2013;2013

17. Wendel CS, Mohler MJ, Kroesen K, Ampel NM, Gifford AL, Coons SJ (2001) Barriers to Use of Electronic Adherence Monitoring in an HIV Clinic. Annals of Pharmacotherapy 35(9):1010-1015

18. Stringer KL, Azuero A, Ott C et al (2019) Feasibility and acceptability of real-time antiretroviral adherence monitoring among depressed women living with HIV in the deep south of the US. AIDS Behav 23(5):1306-1314

19. Checchi KD, Huybrechts KF, Avorn J, Kesselheim AS (2014) Electronic medication packaging devices and medication adherence: a systematic review. JAMA 312(12):1237-1247

20. McCowan C, Shearer J, Donnan PT et al (2008) Cohort study examining tamoxifen adherence and its relationship to mortality in women with breast cancer. Br J Cancer 99(11):1763-1768

\section{Tables}

Table 1

Sample sociodemographic and medical characteristics 


\begin{tabular}{|c|c|}
\hline \multicolumn{2}{|l|}{ Sociodemographic characteristics } \\
\hline Female & $100.00 \%(88 / 88)$ \\
\hline Age (in years, Range: 31-81) & $M=58.34(S D=10.37)$ \\
\hline White/Caucasian \& Non-Latina & $92.05 \%(81 / 88)$ \\
\hline Hispanic/Latina & $3.41 \%(3 / 88)$ \\
\hline Biracial & $1.14 \%(1 / 88)$ \\
\hline $\begin{array}{l}\text { Native American/ } \\
\text { Alaskan Native }\end{array}$ & $1.14 \%(1 / 88)$ \\
\hline Black/African American & $0.00 \%(0 / 88)$ \\
\hline Other & $2.27 \%(2 / 88)$ \\
\hline \multicolumn{2}{|l|}{ Cancer treatment history } \\
\hline $\begin{array}{l}\text { Months from end of primary treatment } \\
\text { (Range 1-36) }\end{array}$ & $M=13.83(S D=8.54)$ \\
\hline Months prescribed endocrine therapy & $M=13.11(S D=8.29)$ \\
\hline \multicolumn{2}{|l|}{$\%$ who received: } \\
\hline $\begin{array}{l}\text { 1. Surgery } \\
\text { 2. Chemotherapy } \\
\text { 3. Targeted Therapy } \\
\text { 4. Radiation }\end{array}$ & $\begin{array}{l}\text { 1) } 100.00 \%(88 / 88) \\
\text { 2) } 34.09 \%(30 / 88) \\
\text { 3) } 10.23 \% \quad(9 / 88) \\
\text { 4) } 68.18 \%(60 / 88)\end{array}$ \\
\hline \multicolumn{2}{|l|}{ Endocrine Therapy Type: } \\
\hline $\begin{array}{l}\text { 1. Aromatase Inhibitors } \\
\text { 2. Tamoxifen }\end{array}$ & $\begin{array}{l}\text { 1) } 62.50 \%(55 / 88) \\
\text { 2) } 37.50 \%(33 / 88)\end{array}$ \\
\hline \multicolumn{2}{|l|}{ Cancer stage } \\
\hline 0 & $5.68 \% \quad(5 / 88)$ \\
\hline I & $71.59 \%(63 / 88)$ \\
\hline II & $18.18 \%(16 / 88)$ \\
\hline III & $4.55 \% \quad(4 / 88)$ \\
\hline
\end{tabular}

Table 2

Patient suggestions from semi-structured exit interviews for Wisepill use 


\section{Daily log}

Participants wanted access to an online or appbased daily medication log that they could check themselves as often as they wanted.
"It might also be beneficial if you could go online and, say you forget, gosh did I take my medication? When did I skip it, or when did I take it, kind of a thing? And if you could go online and get that data on a daily basis from the Wisepill that would be pretty handy."

\section{Reminders}

Participants wanted a reminder (e.g., alarm, blinking light, text message) from the Wisepill to remind them to take their medication.
"If there was some mechanism that would say you haven't taken your pill within twenty-four hours...It would just be more of some type of reminder that yes, you have taken your pill or no you haven't."

\section{Pillbox divider}

Participants wished that the Wisepill had a weekly divider with the days of the week separated to help them keep track of their medications.
"I think sometimes for people taking prescriptions, that it is helpful to have almost a Sunday, Monday, Tuesday, Wednesday, Thursday, Friday type container because at least for me, it's easy to forget once l've gotten up in the morning to get involved with something and then forget whether or not l've taken my medication." 